\title{
Genome-wide investigation of the ZF-HD gene family in Tartary buckwheat (Fagopyrum tataricum)
}

\author{
Moyang Liu ${ }^{1,2+}$, Xiaoxiang Wang ${ }^{1 \dagger}$, Wenjun Sun ${ }^{1}$, Zhaotang Ma', Tianrun Zheng ${ }^{1}$, Li Huang ${ }^{1}$, Qi Wu', \\ Zizhong Tang ${ }^{1}$, Tongliang Bu${ }^{1}$, Chenglei $\mathrm{Li}^{1}$ and Hui Chen ${ }^{1 *}$ (D)
}

\begin{abstract}
Background: ZF-HD is a family of genes that play an important role in plant growth, development, some studies have found that after overexpression AtZHD1 in Arabidopsis thaliana, florescence advance, the seeds get bigger and the life span of seeds is prolonged, moreover, ZF-HD genes are also participate in responding to adversity stress. The whole genome of the ZF-HD gene family has been studied in several model plants, such as Arabidopsis thaliana and rice. However, there has been little research on the ZF-HD genes in Tartary buckwheat (Fagopyrum tataricum), which is an important edible and medicinal crop. The recently published whole genome sequence of Tartary buckwheat allows us to study the tissue and expression profiles of the ZF-HD gene family in Tartary buckwheat on a genome-wide basis.
\end{abstract}

Results: In this study, the whole genome and expression profile of the ZF-HD gene family were analyzed for the first time in Tartary buckwheat. We identified 20 FtZF-HD genes and divided them into MIF and ZHD subfamilies according to phylogeny. The ZHD genes were divided into 5 subfamilies. Twenty FtZF-HD genes were distributed on 7 chromosomes, and almost all the genes had no introns. We detected seven pairs of chromosomes with fragment repeats, but no tandem repeats were detected. In different tissues and at different fruit development stages, the FtZF-HD genes obtained by a real-time quantitative PCR analysis showed obvious expression patterns.

Conclusions: In this study, 20 FtZF-HD genes were identified in Tartary buckwheat, and the structures, evolution and expression patterns of the proteins were studied. Our findings provide a valuable basis for further analysis of the biological function of the ZF-HD gene family. Our study also laid a foundation for the improvement of Tartary buckwheat crops.

Keywords: Tartary buckwheat, ZF-HDs, Genome-wide, Fruit development, Expression patterns

\section{Background}

Specialized genetic networks regulate plant growth by encoding various proteins. Proteins containing transcription factors that bind to specific nucleotide sequences play an important role in different stages of plant growth, flowering, fruiting, and resistance to stress [1, 2]. A homeodomain (HD), as an NDA domain (BD), has 60 conserved amino acid sequences and encodes a homeobox $(\mathrm{HB})$ gene in all eukaryotic transcription

\footnotetext{
*Correspondence: chenhui@sicau.edu.cn

${ }^{+}$Moyang Liu and Xiaoxiang Wang contributed equally to this work.

'College of Life Science, Sichuan Agricultural University, Ya'an, China

Full list of author information is available at the end of the article
}

factors [3]. The 60 amino acids of the homeodomain fold into a characteristic three-helix structure called recognition helix, which attaches to the main sulcus of DNA to form a special connection to DNA $[4,5]$. The HD protein is involved in the development of plants and animals by regulating the expression pattern of target genes [3]. Most HD proteins are related to the proteinprotein interactions and other domains/motifs with regulatory functions [6]. Proteins with homologous domains are divided into six different families according to different themes: leucine zipper-associated HD (HDZip), zinc finger motif-associated HD (ZF-HD), WUSCHEL-related homeobox (WOX), Bell-type HD,

(c) The Author(s). 2019 Open Access This article is distributed under the terms of the Creative Commons Attribution 4.0 International License (http://creativecommons.org/licenses/by/4.0/), which permits unrestricted use, distribution, and 
finger-like domain associated with an HD (PHD finger), and knotted-related homeobox (KNOX) proteins [7].

The zinc finger structure is an important structure and is composed of zinc ions and cysteine or histidine (in most cases) [8]. The zinc finger, as an important motif, is widely found in a variety of regulatory proteins, can specifically bind to DNA/RNA sequences, and participates in protein interactions $[9,10]$. Zinc finger units are divided into many categories according to Cys and differences in residues, such as $\mathrm{C} 3 \mathrm{hC} 2 \mathrm{H} 2$ and $\mathrm{C} 2 \mathrm{C} 2$ [8]. As a family, the zinc finger homeodomain (ZF-HD) proteins, containing $\mathrm{HD}$ proteins and a zinc finger related to the homeodomain, were first identified in the $\mathrm{C} 4$ plant Flaveria [11].

At present, the $Z F-H D$ gene family has been identified in Arabidopsis thaliana, rice (Oryza sativa) and tomato (Solanum lycopersicum) [12-14]. Arabidopsis thaliana has 17 ZF-HD members, and they act as transcription factors, have unique physiological characteristics, and play a very important role in the development of flowers [11]. Drought, salinity and abscisic acid (ABA) can induce AtZHD1 to bind to the ERD1 promoter region [15] Three genes were found in the $Z F-H D$ gene family of Arabidopsis thaliana, and its mini zinc finger (MIF) gene and protein sequence encoded by congeners are highly similar to the ZF domain of the ZF-HD protein but have no HD domain $[12,16]$. The phylogeny and sequence analyses of MIF and $Z H D$ genes were conducted, suggesting that the $Z H D$ gene has plant specificity and that almost all genes have no introns [11]. Until now, the origin and evolution of the ZHD and MIF gene populations remain unclear [12]. In soybean, GmZF-HD1 and GmZF-HD2 bind to the promoter region of the gene encoding calmodulin subtype 4 ( $G m C a M 4)$, and the expression of GmZF-HD1 and GmZF-HD2 increased after inoculation with pathogenic bacteria [17]. In tomato, the $Z F-H D$ gene family was found to be related to fruit development as well as to stress [14]. The $Z F-H D$ gene family may play a similar role in Tartary buckwheat.

Common buckwheat (Fagopyrum esculentum) is produced in southwestern China and has spread to all continents. Tartary buckwheat (Fagopyrum tataricum) grows in the mountainous areas of southwestern China, northern India, Bhutan and Nepal [18]. Tartary buckwheat is currently the only widely grown single-sex only food crop, has a balanced essential amino acid composition in its seed protein, and has a total protein content that is higher than that in many food crops $[8,19]$. The $Z F-H D$ gene family has been widely studied in many plants, some studies have found that after overexpression AtZHD1 in Arabidopsis thaliana, florescence advance, the seeds get bigger and the life span of seeds is prolonged [12, 20], moreover, $Z F-H D$ genes are also participate in responding to adversity stress [21]. However, the $Z F-H D$ gene family in Tartary buckwheat has not been studied, at present, the ARF, AP2, NAC, MADS genes family have been studied deeply in Tartary Buckwheat [22-25]. Because of the important role of the $Z F$ $H D$ gene in various physiological processes, it is of great significance to systematically study the Tartary buckwheat ZF-HD family. The recently completed genome sequence of Tartary buckwheat provides an opportunity to reveal the tissue, expression and evolution characteristics of the $Z F-H D$ gene family in Tartary buckwheat at the whole genome level. In this paper, the exon-intron structure, motif composition, genomic structure, chromosome location, sequence homology and expression pattern of 20 Tartary buckwheat ZF-HD genes are introduced in detail. In addition, the phylogenetic relationship between the ZF-HD gene family in Arabidopsis thaliana and Tartary buckwheat was compared. Through global expression analysis, the degree of participation of ZF-HD gene family members in different biological processes of Tartary buckwheat was determined. The role of the FtZF-HD gene in the development of buckwheat fruit was studied in detail, which provided a valuable clue for the functional characterization of the buckwheat $Z F-H D$ gene family members in the growth and development of Tartary buckwheat.

\section{Results}

Identification of the FtZF-HD genes in Tartary buckwheat

To identify the FtZF-HD genes in Tartary buckwheat, all possible FtZF-HD members in the Tartary buckwheat genome were mined using two BLAST methods, multiple FtZF-HD genes from the Tartary buckwheat genome were isolated by these two methods, and since the buckwheat genome was sequenced using a genome-wide shotgun strategy, some of these FtZF-HD genes may be redundant even though they were on different scaffolds. In this study, we identified a total of $20 Z F-H D$ genes, and we named them FtZHD1 FtZHD17 and FtMIF1 FtMIF3 based on their physical location on the chromosomes (Additional file 2: Table S1).

For the Tartary buckwheat FtZF-HD proteins, FtZHD14 was the smallest protein with 83aa, and the largest protein was FtZF-HD5 (330aa) (Additional file 2: Table S1). The molecular weight of the proteins ranged from $9.33 \mathrm{kda}$ to $34.95 \mathrm{kda}$; PI from 4.94 (FtMIF1) to 10.15 (FtZHD10). The predicted subcellular localization results showed that all proteins are located in the nuclear region.

\section{Phylogenetic analysis of the ZF-HD gene family in Tartary buckwheat}

To investigate the phylogenetic relationship of the ZFHD proteins in Tartary buckwheat, the neighbor-joining 
(NJ) method of Geneious R11 was used to construct a phylogenetic tree consisting of Arabidopsis thaliana (17 gene) and Tartary buckwheat (20 gene). The phylogenetic distribution indicated that the FtZF-HD gene family can be divided into two subfamilies: MIF and $Z H D$. $Z H D$ was further divided into five parts (ZHDI, ZHDII, ZHDIII, ZHDIV and ZHDV) (Fig.1). The number of $Z H D I V$ genes was the least (5\%) and that in the ZHD II gene family was the highest (35\%). In addition to FtZHD17 and FtZHD12, the ZF-HD gene family of Tartary buckwheat was similar to the ZF-HD family of Arabidopsis thaliana; the highest degree of similarity was $78.85 \%$ (FtZHD14), and the lowest was $38.06 \%$ (FtZHD9). FtZHD17 and FtZHD12 were more similar to rice OsZHD1, 44.71 and 49.27\%, respectively (Additional file 2: Table S1). Moreover, the results showed that the ZF-HD gene was amplified in Tartary buckwheat.

\section{Structural analysis and motif composition of the Tartary buckwheat ZF-HD gene family}

To further understand the structural components of the FtZF-HD gene, we compared the corresponding genomic DNA sequences, and we obtained the exon and intron structures of the FtZF-HD genes (Fig. 2b). Interestingly, most of the FtZF-HD genes (16 out of 20) did not have introns, but two of the other four genes did have an intron. The remaining two genes had two introns each. Moreover, the location of the intron of the two genes was very close, and there may be a replication relationship between the two introns. Most genes with similar evolutionary relationships have similar exonintron structures. When genes do not have introns, it means that they are not easily connected and have relatively conserved functions. This result is consistent with the result from the previously reported case in which the $Z F-H D$ gene had almost no introns [11]. The intron of the Tartary buckwheat $Z F-H D$ gene is inherited via evolution or obtained by mutations during evolution, and whether the appearance of introns has an effect on the original function of these genes is a question that needs to be further studied.

To further study the characteristic region of the FtZF$H D$ protein, the structures of $20 \mathrm{FtZF-HD}$ proteins were analyzed by online MEME analysis. According to the

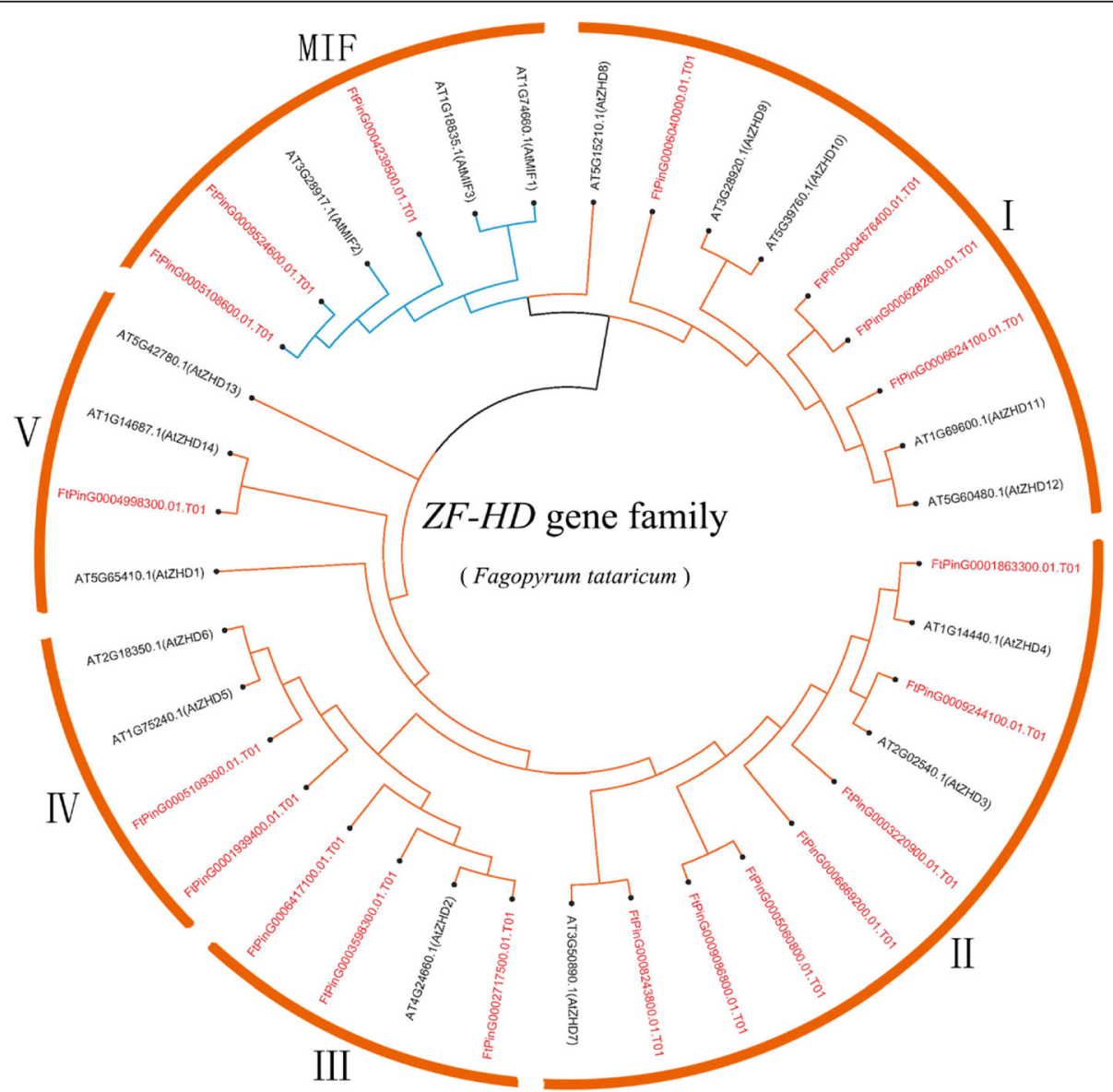

Fig. 1 Unrooted phylogenetic tree based on the relationship between Tartary buckwheat and Arabidopsis. Each arc represents a different group (or subgroup) of ZF-HD 


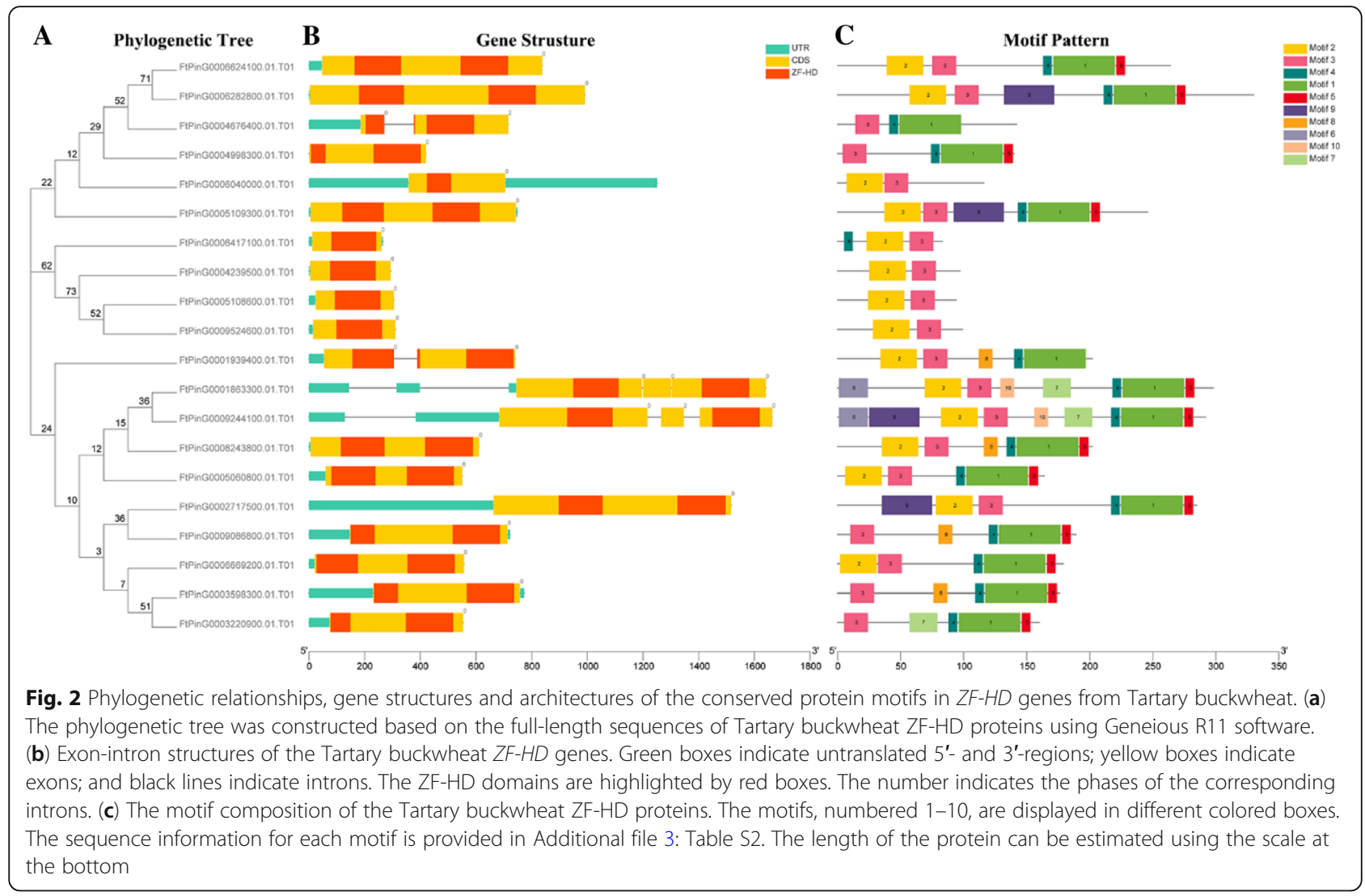

results of the MEME analysis, a schematic map was constructed to characterize the structure of the FtZF-HD protein. We identified 10 conserved motifs, named motifs 1 to 10 (Fig. 2c). It is worth noting that all FtZF-HD genes had motif3 domains, and most (60\%) had motif2 domains. Interestingly, motif4 was a domain that was specific to the ZHD family, and motif1 and motif5 were detected in almost all ZHD genes. Motif6 and motif10 were detected specifically in the FtPinG0001863300.01 and FtPinG0009244100.01genes. Motif8 and motif9 existed in four genes each. Motif7, motif8, and motif9 only existed in a few genes. Compared with the MIF gene family, the $Z H D$ gene family showed obvious differences, and the functional differences in the $Z H D$ gene in Tartary buckwheat were probably due to the subfamilyspecific distribution of conserved motifs. The same motifs in three MIF genes and the same motifs in several subpopulations of $Z H D$ indicated that there were conserved motifs in the subpopulations, but the function of these conserved motifs remains to be clarified.

\section{Chromosome distribution and synchronous analysis of the FtZF-HD genes}

There was an uneven distribution of the FtZF-HD genes on 7 buckwheat chromosomes (except Ft2). Chromosome 3 and chromosome 7, which were the two chromosomes with the most ZF-HD genes, each contained four $Z F-H D$ genes, and three chromosomes had the lowest number of $Z F-H D$ genes (2 ZF-HD genes) (Fig. 3). Gene replication plays an important role in the occurrence of new functions and gene amplification. To determine the fragment replication events between the genes, we adopted the standard [26]. When the query coverage and consistency of the candidate genes are $\geq 80$, they are thought to be repetitive genes. Chromosomal regions within the $200 \mathrm{~kb}$ range of two or more genes are defined as tandem replication events. Therefore, the analysis of Tartary buckwheat gene duplication showed that no tandem repeat gene was found on the chromosomes of Tartary buckwheat; however, 12 genes were involved in fragment repeat events, and the ZF-HD gene on chromosome 4 was involved in the most fragment repeat events (Fig. 3). These results suggest that some FtZF-HD genes may be produced by repeated fragments of the gene, and these replication events are the main driving force in the evolution of FtZF-HD.

To further infer the phylogenetic relationship between Tartary buckwheat and dicotyledonous plants, we analyzed the collinear relationships between seven plants and Tartary buckwheat (Fig. 4). The results showed that 23 FtZF-HD genes were collinear with soybean genes, followed by grape (15), tomato (13), 


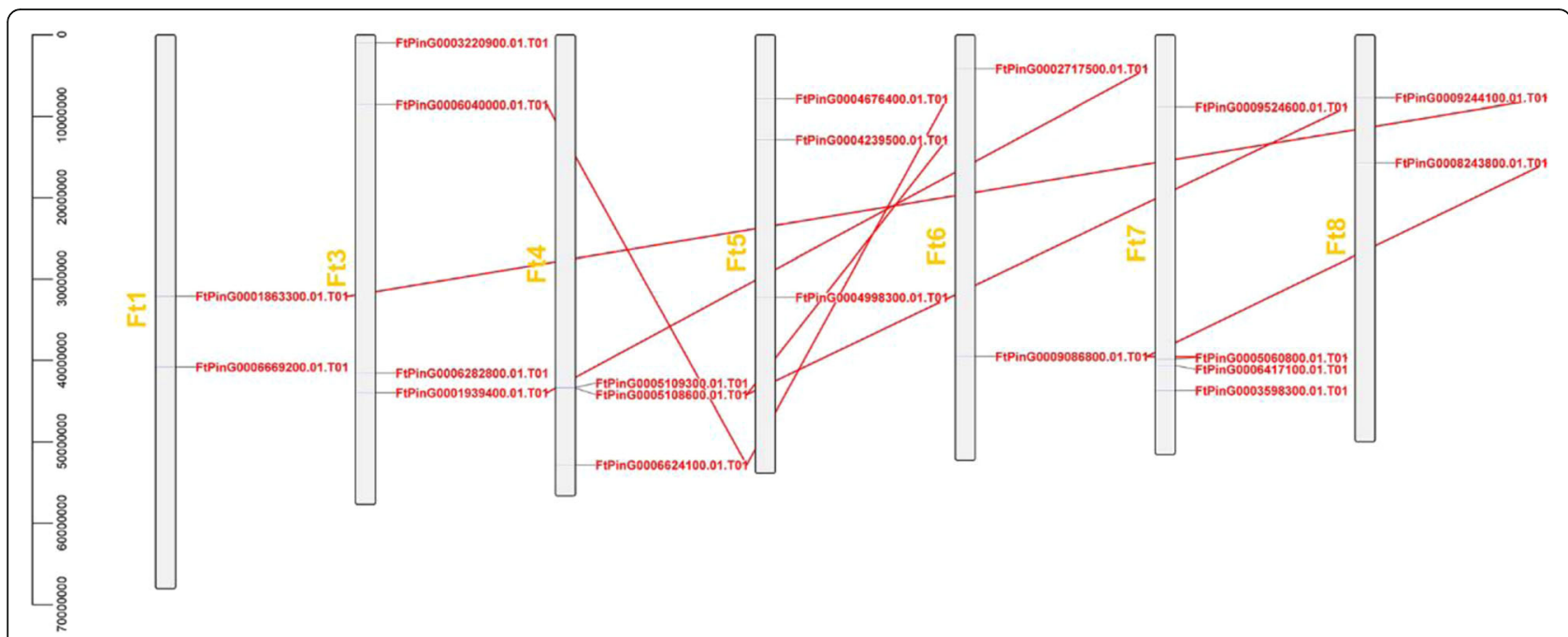

Fig. 3 Schematic representations of the chromosomal distribution and inter-chromosomal relationships of the Tartary buckwheat ZF-HD genes. The red color denotes the ZF-HD gene, and the red line indicates that there is a fragment repetition of the two genes. The chromosome number is marked in yellow

beet (9), sunflower (6), Arabidopsis (5), and rice (4). Some FtZF-HD genes were associated with at least two pairs of homologous genes, especially the ZF-HD genes of Tartary buckwheat and soybean, such as FtPinG0006040000.01, FtPinG0006624100.01and FtPin G0002717500.01, which may play an important role in the evolution of the ZF-HD gene family (Additional file 4: Table S3). The relationships between Tartary buckwheat and soybean, tomato, sugar beet and grape were similar. The comparison of the ZFHD gene analysis between Tartary buckwheat and other plants is of great significance for establishing the genetic relationship among species and predicting the function of genes.

\section{Evolution analysis of the FtZF-HD gene family within several different species}

The number of FtZF-HD genes identified was similar to that of Arabidopsis thaliana (17), rice $(15,14]$ and other plant $Z F-H D$ gene families. But the genome size of the three species was very different (Tartary buckwheat, 516 $\mathrm{Mb}$; Arabidopsis thaliana, $125 \mathrm{Mb}$; rice, $466 \mathrm{Mb})$. This result indicated that the $Z F-H D$ gene family remained stable in different species during long-term evolution. Based on the existing ZF-HD genes of Tartary buckwheat, the replication and diversity of the $Z F-H D$ genes during evolution were further studied. We constructed a phylogenetic tree with the $Z F-H D$ protein sequences of Tartary buckwheat, a monocotyledonous plant (rice) and six dicotyledonous plants (Arabidopsis thaliana, soybean, tomato, beet, grape and sunflower) using the neighbor-joining method of Geneious R11. The ZF-HD proteins were divided into five groups according to the phylogenetic tree (Fig. 5). Group A contained the most $Z F-H D$ genes (7 genes) in Tartary buckwheat, Group C and Group D contained 4 each, and Group Band Group $\mathrm{E}$ contained the least (2 genes).

We also use MEME web servers to search for conserved motifs shared by the ZF-HD proteins. The results showed (Fig. 5) that 10 different conserved motifs were identified. The genes of the same group had similar motifs, such as those in group $d$, which suggests potential functional similarities among the ZF-HD proteins. Motif 1 and motif 4 were conserved motifs shared by almost all ZF-HD proteins, indicating that ZF-HD had some highly conserved domains. Motif2 and motif 3 were conserved domains shared by almost all ZF-HD proteins except for group $\mathrm{d}$; therefore, group $\mathrm{d}$ may have evolved from other genes after the loss of motif2 and motif3 or other genes evolved after acquiring motif2 and motif3.

\section{Expression patterns of the FtZF-HD genes in different plant tissues}

To study the physiological function of the FtZF-HD genes, a real-time PCR technique was used to detect the time of expression of individual members of the gene family. The accumulation of FtZF-HD transcription products in the roots, stems, leaves, flowers, fruits and other tissues of Tartary buckwheat was evaluated (Fig. 6) . The results showed that the transcriptional abundance of the FtZF-HD gene varied greatly in the different tissues and organs, suggesting that the FtZF-HD gene family has many functions in the growth and development of Tartary buckwheat. Some FtZF-HD genes that were expressed in Tartary buckwheat stem had tissue specificity, gene FtMIF3 was expressed only in flowers, 

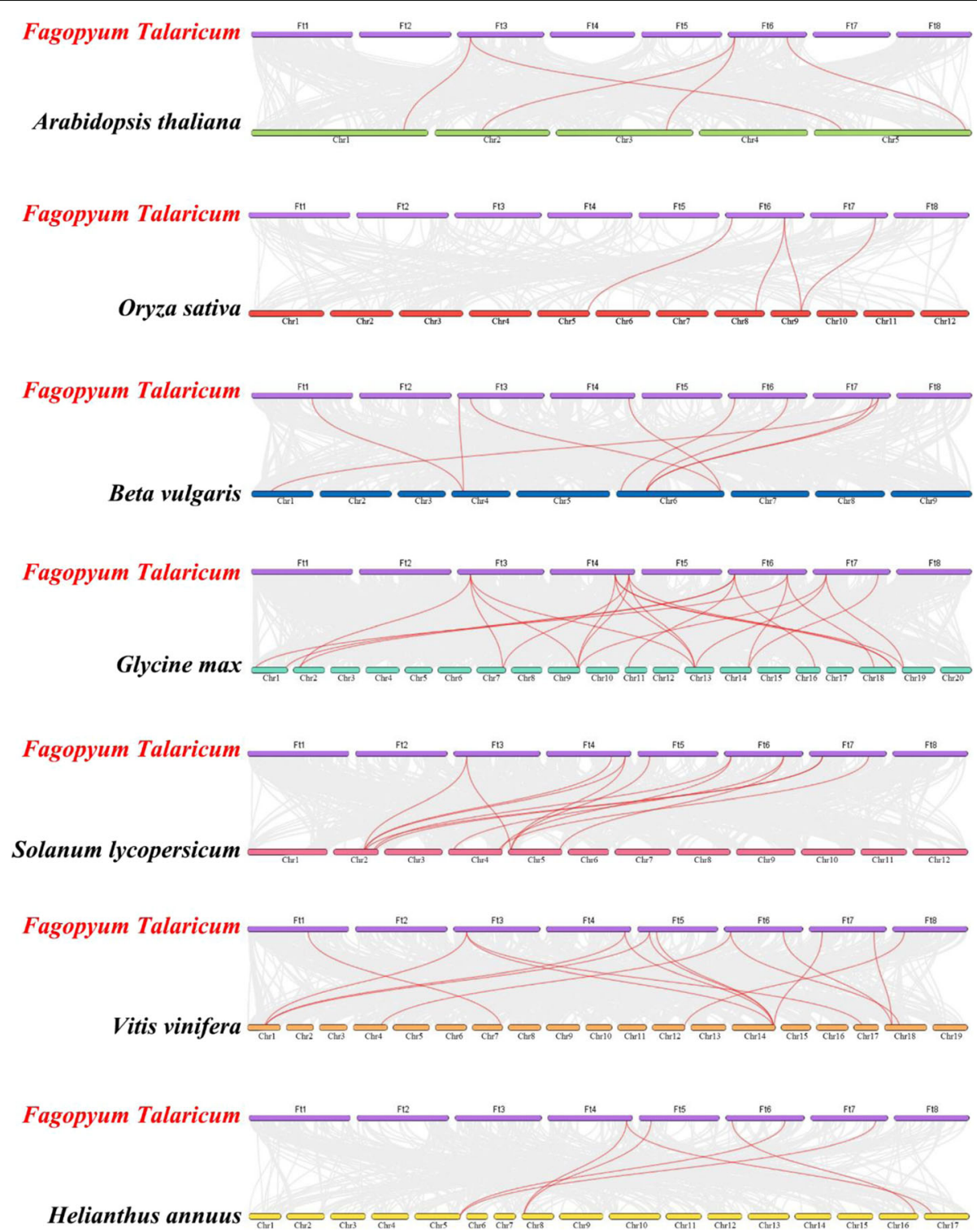

Fig. 4 Synteny analysis of the ZF-HD genes between Tartary buckwheat and seven representative plant species. Gray lines in the background indicate the collinear blocks within the Tartary buckwheat and other plant genomes, while the red lines highlight the syntenic ZF-HD gene pairs

FtZHD10 and FtZHD3 were expressed only in the roots, FtZHD2 was only not expressed in the fruit, and FtZHD13 and FtMIF1 were not expressed in leaves. Four FtZF-HD genes (FtZHD11/ FtZHD6/ FtZHD15/ FtZHD13) in Tartary buckwheat fruit had high levels of expression. The expression of 11 FtZF-HD genes (FtZHD1/2/4/5/7/9/12/16/17 and FtMIF2/3) in the flowers was higher than that in other organs. This study found that only five FtZF-HD genes were expressed more in the reproductive organs than in the other tissues (FtZHD1/6/11/12/15) (Fig. 6).

We also studied the correlations among the FtZF-HD gene expression patterns in the roots, stems, flowers, leaves and fruits of Tartary buckwheat. Multiple pairs of genes had a strong correlation (0.880-0.986): FtZHD 17 and FtMIF3, FtZHD 17 and FtZHD 16, FtZHD 17 and FtZHD4, FtZHD 11 and FtZHD 15, FtZHD 9 and FtZHD 5, FtZHD 5 and FtZHD 4, FtZHD 4 and FtMIF3, FtZHD 4 and FtZHD 2, and FtZHD 1 and FtZHD 12 (Fig. 7).

\section{Differential expression of the FtZF-HD genes during fruit} development of Tartary buckwheat

To further study the expression of the FtZF-HD gene in buckwheat fruit, real-time PCR was used to detect the expression of the members of the gene family in the three periods of fruit development (13 days after pollination (DAP), 19 DAP and 25 DAP) [27] and to evaluate the accumulation of FtZF-HD transcription products in 


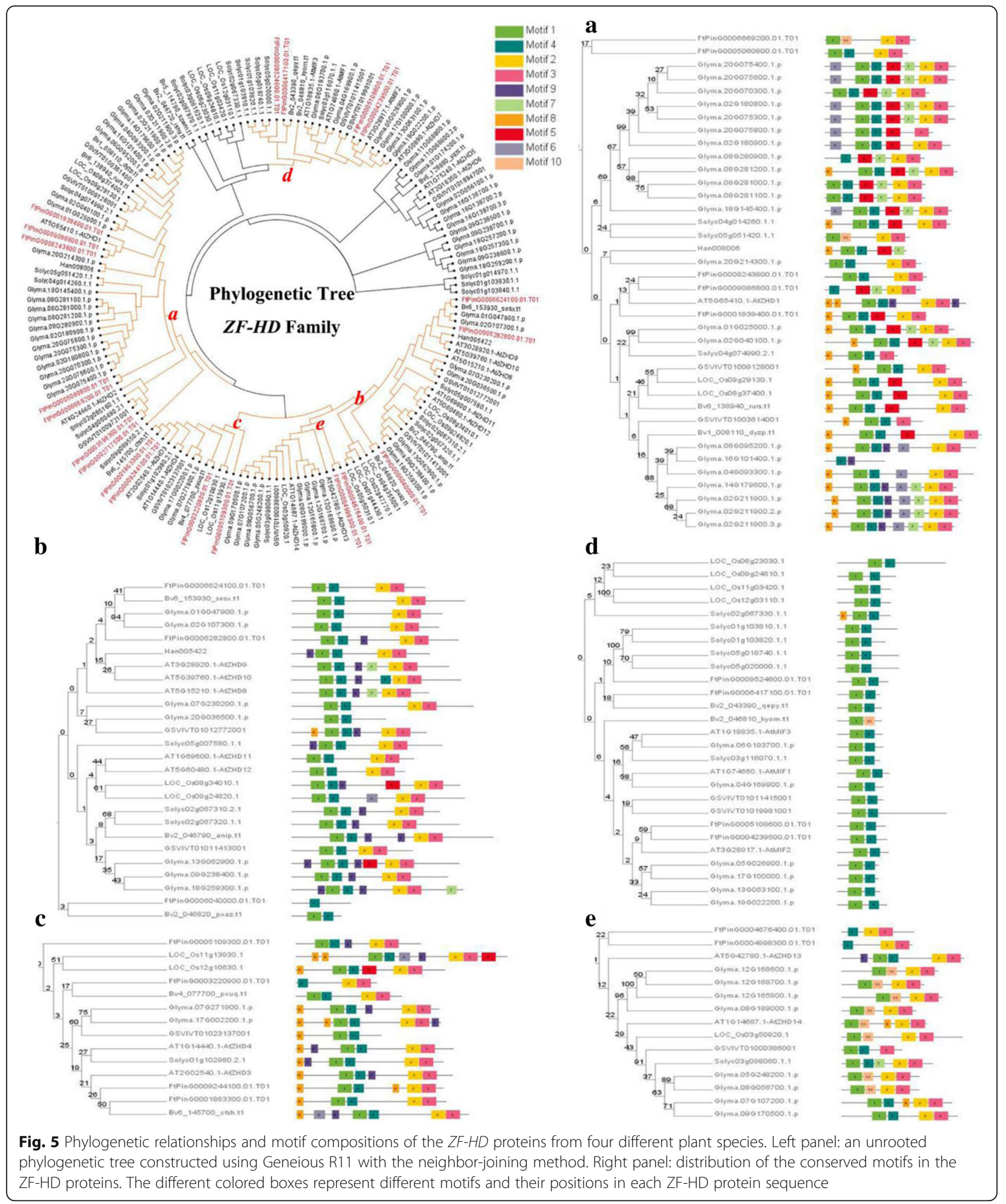

buckwheat fruits (Fig. 8). The results showed that most of the FtZF-HD genes were highly expressed in the first period (13 DAP) of fruit development, implying their contribution in the early stages of fruit development. The expression of 5 FtZF-HD genes in the third period (25 DAP) of fruit development was higher than that in 


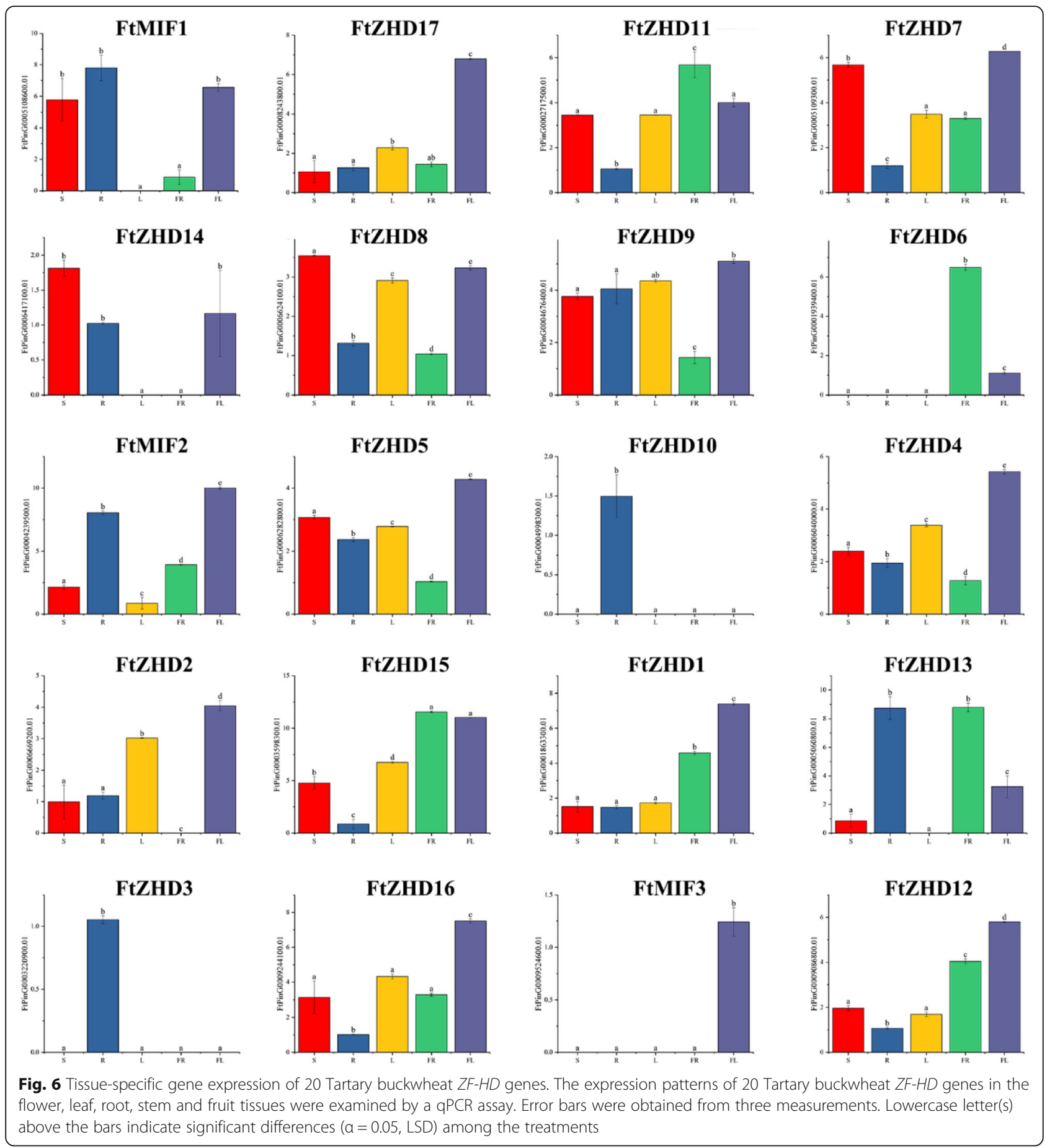

other periods, which implies their contribution to fruit ripening. The expression of all FtZF-HD genes in the fruit during the second period (19 DAP) was not higher than that during the other two periods, although some of the FtZF-HD genes were highly expressed during the second period. In this study, only one FtZF-HD gene (FtPinG0006624100.01) was highly expressed during all three periods of fruit development, and the level of expression was stable (Fig. 8).

At the same time, we also studied the correlations among the expression patterns of the FtZF-HD genes in three stages of fruit development. Three pairs of genes had strong correlations (0.998-1.000): FtMIF1 and FtMIF2, FtZHD17 and FtZHD12, and FtZHD12 and FtZHD1 (Fig. 9). 


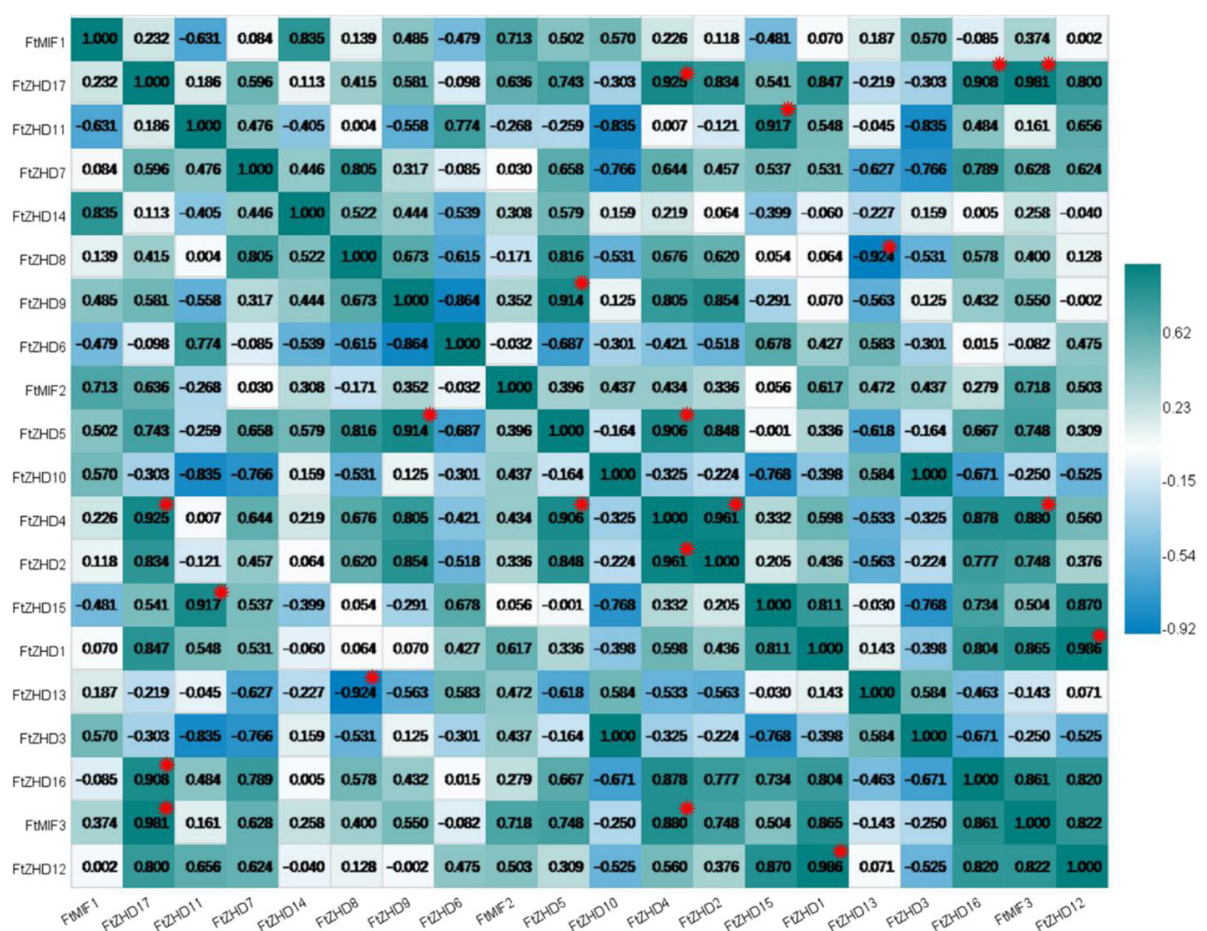

Fig. 7 The correlation between the gene expression patterns of FtZF-HDs. Green: positively correlated; Blue: negatively correlated. *(red) indicates a significant correlation at the 0.05 level

\section{Discussion}

A phylogenetic analysis and sequence analysis of the $Z H D$ and MIF genes indicate that both genes are endemic to terrestrial plants and belong to two different groups of the same protein family (ZF-HD). Extensive research has shown that $Z H D$ has been found in many terrestrial plants but not in algae [12]. We identified 20 ZF-HD genes in Tartary buckwheat, including 17 ZHD genes and 3 MIF genes, which was slightly higher than the number found in Arabidopsis (17). The replication of genes can amplify the number of genes. For example, four large replication events occurred in the Arabidopsis genome, and more than half of $Z F-H D$ genes may be produced by genomic replication [12, 28, 29]. Gene replication mechanisms include fragment replication, tandem replication and translocation (reverse transcription and replication translocation), which is an important factor in biological evolution [30]. Among the mechanisms, fragment replication is one of the main contributors to the amplification of many gene families [31]. The analysis of the chromosome distribution of the ZF-HD genes in Tartary buckwheat revealed there was fragment replication, but no tandem replication, which suggests that the gene fragment replication events greatly facilitates the expansion of the $Z F-H D$ gene family in plants with smaller genomes. Moreover, a phylogenetic tree with Arabidopsis also suggests an evolutionary relationship between these two species. There are three main mechanisms for the variation of the exon and intron structures of a gene (the gain or loss, exonization or pseudoexonization and insertion or deletion of exon or intron), and each mechanism leads to a gene structural difference $[15,32-34]$. The presence of a few introns in the Tartary buckwheat ZF-HD gene may be due to the variations in the participation of these three mechanisms. To study the phylogenetic relationship between Tartary buckwheat and dicotyledon, we constructed collinear relationship maps between Tartary buckwheat and seven plant species (Fig. 4). Finally, 23 colinear $Z F-H D$ gene pairs of Tartary buckwheat and soybean were identified. The number of orthologous events was far greater than that between Tartary buckwheat and rice, which was consistent with the closer evolutionary distance between Tartary buckwheat and soybean [35].

The ZF-HD transcription factors are involved in various biological processes such as the response of plants to abiotic stress and the development of plants by phytohormones [34, 36]. Most of the previous reports on the $Z F-H D$ gene were about the regulation of the ZF-HD gene in response to abiotic stress, but there were few reports on plant development. Members of the ZF-HD gene family are expressed in floral tissues in plants (e.g. Arabidopsis) [36]. It was revealed that the $Z F-H D$ gene family regulates flower development. Our research shows that the 20 ZF-HD genes identified from Tartary 


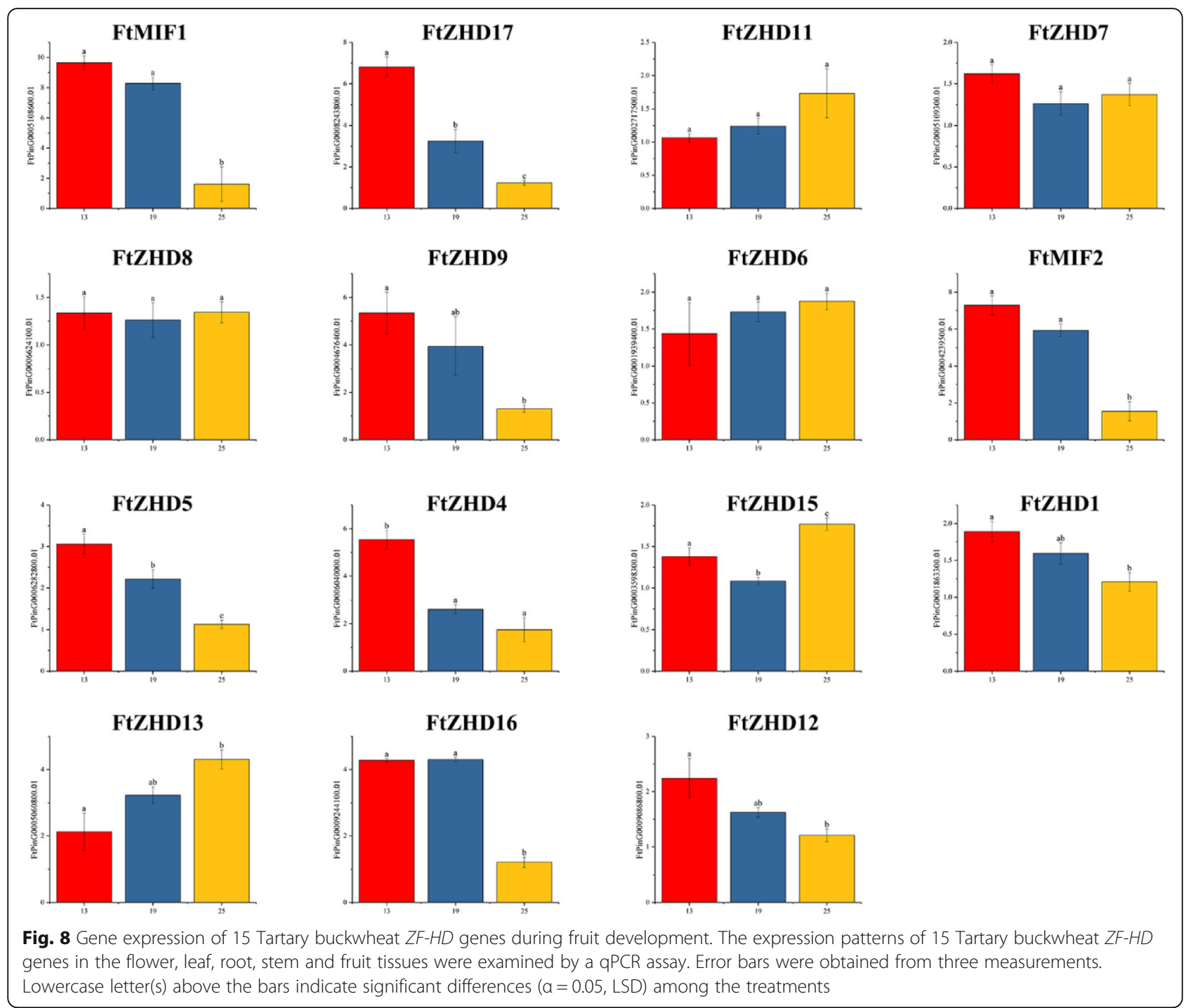

buckwheat were indeed expressed during the growth and development of plants, so there is no pseudogene. However, in the study of fruit development, we found that five genes were not involved in the regulation of fruit development. It is worth noting that FtMIF3, which belongs to the same subfamily as FtMIF1 and FtMIF2, is not expressed in the fruit, and when the motifs of the three were compared (Fig. 2), we found that they had the same motifs. This result leads us have great interest in exploring the reasons for the differences among the genes because after comparing their protein sequences (Additional file 1: Figure S1), we found that although the three motifs are the same, the amino-acid residues at the 126th to 139th positions of the amino acid sequence encoded by the FtMIF3 gene differ significantly from those of FtMIF1 and FtMIF2. We speculate that it is highly likely that a mutation in the FtMIF3 gene caused the protein it encodes to lose its ability to express itself in the fruit. For FtMIF1 and FtMIF2, their differences in amino acid sequences have little effect, and the expression of FtMIF1 and FtMIF2 in fruit development has a 0.998 correlation. Although not all genes are involved in fruit development, it is equally exciting to find that 15 genes are expressed in the fruit. Before this study, the expression of the ZF-HD protein family in fruit was only reported in grape (Vitis vinifera) and tomato, and there was only a potential role in grape fruit development $[14,15]$.

We found four grape genes that were expressed in fruits in the phylogenetic trees containing multiple plants (Fig. 5) (GSVIVT01003614001, GSVIVT01009128001, GSVIV T01012772001 and GSVIVT 01011413001, in groups a and b, respectively) [15]. These genes have similar to the motifs of those of Tartary buckwheat in the respective groups. For example, the genes in group a have motif1 and motif3, and the genes, except for FtPinG 006040000.01, in group b have motif1, motif2, motif3 and 


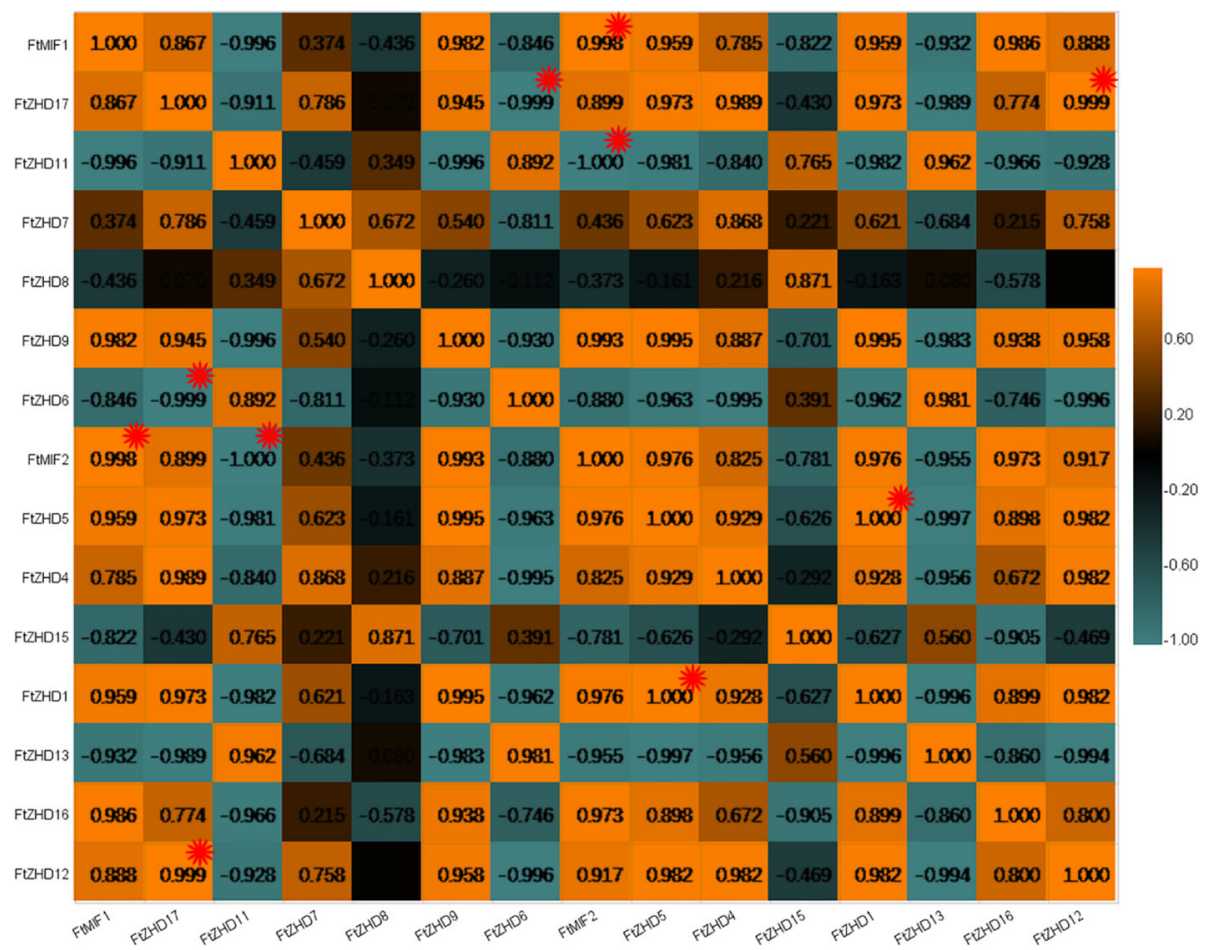

Fig. 9 The correlation between the gene expression patterns of FtZF-HDs during fruit development. Orange: positively correlated; Green: negatively correlated. ${ }^{*}($ red) indicates a significant correlation at the 0.05 level

motif4. It can be seen that the relationship between the grape genes and Tartary buckwheat genes in group $\mathrm{b}$ is closer than that in group a.

In analyzing the expression of genes in the tissues (Fig. 6), we noted that five genes are more highly expressed in reproductive organs (flowers and fruits) than in other tissues (FtZHD1, FtZHD6, FtZHD12, FtZHD15, and FtZHD11). In addition, the expression of FtZHD1 and FtZHD12 in the flowers was higher than that in the fruit, and the expression of FtZHD6, FtZHD15 and FtZHD11 in the fruit was higher than that in the flowers. Comparing their motifs (Fig. 2), we found that FtZHD12 and FtZHD15 have exactly the same motifs. Therefore, we speculate that there may be a functional overlap between the two genes. With the study of their expression during fruit development (Fig. 8), we found that the expression of FtZHD12 and FtZHD1 in the earlier stage was higher than that in the middle and late stages, the expression of FtZHD6, FtZHD11 and FtZHD15 in late stage was higher than that in middle stage and earlier stage, and it was discovered by a synchronous analysis of the genes (Fig. 3) that there was fragment duplication between FtZHD6 and FtZHD11. Therefore, FtZHD6 and FtZHD11 have similar functions in the development of the reproductive organs and fruits. We also found that FtZHD11 and FtZHD15 have a
0.917 correlation in plant tissue development, so we speculate that FtZHD11 and FtZHD15 may also have functional overlaps.

The size of monocotyledonous fruit is related to endosperm development [37, 38], in dicotyledonous plants, the final size of the fruit is related to the number and size of cotyledon cells [39]. Studies have pointed out that usually the initial growth of the endosperm rather than the late growth of the embryo is mainly related to the size of the fruit [40-42]. Reports on the development of Tartary buckwheat fruit indicated that the size of Tartary buckwheat fruit is mainly related to cell division during embryonic development, and most Tartary buckwheat fruits reach the maximum state on the 13th to 25th day after pollination. [43]. Therefore, in this study, the early stage of fruit development is the key period in determining the size of the fruit. In our research on fruit development, we found that there are 8 genes that are more highly expressed in the earlier stages than in the other two stages. Fruit development is controlled by various transcriptional regulatory networks. These networks involve transcription factors, for example, members of the ARF family, which can regulate hormones such as AUX and ABA [43]. FtARF 2 may have the ability to integrate signals, thus prolonging the cycle of embryonic development, increasing the cycle of cell division and 
increasing the accumulation of storage materials in the fruit tissue [43]. The relationship between FtMIF1, FtMIF2 and FtZHD17 and the FtARF gene family needs further study; however, FtMIF1, FtMIF2 and FtZHD17 may be related to the fruit size of Tartary buckwheat. In addition, FtZHD12, which has a 0.999 correlation with FtZHD17, may also be related to fruit size. In conclusion, the analysis of FtZF-HD gene expression in the tissues and fruit laid a foundation for breeding new Tartary buckwheat varieties.

\section{Conclusions}

20 FtZF-HD genes were identified in Tartary buckwheat, and the structures, evolution and expression patterns of the proteins were studied. Our findings provide a valuable basis for further analysis of the biological function of the $Z F-H D$ gene family. Our study also laid a foundation for the improvement of Tartary buckwheat crops.

\section{Methods}

\section{Identification of ZF-HD genes in Tartary buckwheat}

We downloaded the Tartary buckwheat genome from the Tartary buckwheat genome project (TBGP; http:// www.mbkbase.org/Pinku1/) [22]. The ZF-HD gene family of Tartary buckwheat was searched by two BLASTP methods. The hidden Markov model (HMM) file corresponding to the ZF-HD_dimer domain (PF04770) was downloaded from the Pfam protein family database (http://pfam.xfam.org/). The existence of ZF-HD_dimer core sequences was verified by PFAM and SMART programs. Finally, 20 genes containing $Z F-H D$ domain were screened from tartary buckwheat genome. By using the tools from the ExPASy website (https://web.expasy.org/compute_pi/), we obtained the sequence length, molecular weight, isoelectric point and subcellular localization of the identified $20 Z F-H D$ proteins.

\section{Sequence analysis}

Using the ZF-HD_dimer domain sequence of the FtZF$H D$ proteins, we used the default parameter ClustalW to compare several protein sequences. To determine the exon-intron structure of the FtZF-HD genes, the predicted coding sequence was compared with the corresponding full-length sequence by the Gene Structure Display Server (GSDS: http://gsds.cbi.pku.edu.cn) online program. Using the MEME online program (http:/ meme.nbcr.net/meme/intro.html) to analyze the protein sequences under the following parameters: the optimum motif width was $6 \sim 200$; the maximum number of motifs was 10.

\section{Chromosomal distribution and gene duplication of FtZF- $H D$ genes}

The method of FtZF-HD genes mapping to the chromosomes of tartary buckwheat was refer to Liu et al. [22]. Analysis of FtZF-HD genes replication events using Multiple collinear scanning toolkits (MCScanX). The syntenic relationship between FtZF-HD genes and ZF-HD genes from selected plants were determined by using the Dual Systeny Plotter software (https://github.com/CJChen/TBtools).

\section{Phylogenetic analysis and classification of the FtZF-HD gene family}

Using ZF-HD protein sequences (Arabidopsis thaliana, maize, rice and soybean) downloaded from the UniProt database (https://www.uniprot.org/) constructed phylogenetic trees. We using the NJ method of Geneious R11 to derived the phylogenetic tree. The parameters were the Jukes-Cantor model, and global alignment with free end gaps.

\section{Plant growth}

This study used the Tartary buckwheat (XiQIAO) germplasm provided by Professor Wang Anhu of Xichang University. We planted materials in the experimental field of the College of Life Sciences of Sichuan Agricultural University (Lat. 29 $97^{\prime} \mathrm{N}, 102^{\circ} 97^{\prime} \mathrm{E}$, Alt. $580 \mathrm{~m}$ ), Ya'an, Sichuan, China [22]. The samples including flower, the fruit from three $(13,19$, and 25 , DAP) different developmental fruit stages, and the stem, root, and leaf of mature tartary buckwheat were collected separately and quickly put into liquid nitrogen and stored at $-80^{\circ} \mathrm{C}$ for further use.

\section{Expression analysis of the FtZF-HD genes using real-time PCR}

Using the Primer3 software (http://bioinfo.ut.ee/primer3/) designed the RT-qPCR primers (Additional file 5: Table S4). Quantitative real-time PCR analysis was used to analyze the identified genes. Using the FtH3 gene as an internal control, the standard RT-qPCR with SYBR Premix Ex Taq II (TaKaRa) was repeated at least three times on a CFX96 Real Time System (BioRad). The data were analyzed by the $2^{-(\Delta \Delta \mathrm{Ct})}$ method, and the relative mRNA expression data were obtained [44].

\section{Statistical analysis}

We used Origin Pro 2018b (OriginLab Corporation., Northampton, Massachusetts, USA) statistics program to analyze all the data, and the means were compared by the least significant difference test (LSD) at significance levels of 0.05 and 0.01 [22]. 


\section{Additional files}

Additional file 1: Figure S1 Alignment of multiple FtZF-HD domain amino acid sequences. Colored amino acids indicate that multiple genes have the same amino acids at the same location. (DOCX $231 \mathrm{~kb}$ )

Additional file 2: Table S1 List of the 20 FtZF-HD genes identified in this study. (XLS $67 \mathrm{~kb}$ )

Additional file 3: Table S2 Analysis and distribution of conserved motifs in Tartary buckwheat ZF-HD proteins. (XLS 31 kb)

Additional file 4: Table S3 One-to-one orthologous relationships between tartary buckwheat and other seven plant species. (XLS $44 \mathrm{~kb}$ )

Additional file 5: Table S4 Primers of sequences. (XLS $30 \mathrm{~kb}$ )

\section{Abbreviations}

At: Arabidopsis thaliana; DAP: Days after pollination; Ft: Fagopyrum tataricum; Gm: Glycine max; HB: Homeobox; HD: Homeodomain; HD-Zip: Leucine zipper-associated HD; KNOX: Knotted-related homeobox; MIF: Mini zinc finger; PHD finger: Finger-like domain associated with an homeodomain; WOX: WUSCHEL-related homeobox; ZF-HD: Zinc finger motif-associated homeodomain

\section{Acknowledgements}

We thank all the colleagues in our laboratory for providing useful discussions and technical assistance. We are very grateful to the editor and reviewers for critically evaluating the manuscript and providing constructive comments for its improvement.

\section{Authors' contributions}

M.-Y.L. planned and designed the research, and analyzed data. X.-X.W. wrote the original manuscript. X.-X.W. and Z.-T.M. determined the expression of genes by qRT-PCR. T.-R.Z. and L.H. identified FtZF-HD family genes and visualized their structures. Q.W. and Z.-Z.T. performed the evolutionary analysis of FtZF-HD genes and several different species. T.-L.B. and C.-L.L. performed FtZF-HD genes chromosome distribution, gene replication and synchronous analysis. W.-J.S. planted and collected plant materials. H.C., M.-Y.L. and W.-J.S. reviewed and edited the manuscript. H.C. supervised the research. M.-Y.L. and X.-X.W. contributed equally. All authors read and approved the final manuscript.

\section{Funding}

Funding this research was supported by the National Natural Science Foundation of China (31500289), and the National Key R\&D Program of China (2018YFD1000706). Funds were used for the design of the study and collection, analysis, and interpretation of data and in writing the manuscript, as well as in the open access payment.

\section{Availability of data and materials}

The genome sequences of Tartary buckwheat used for identifying the ZF-HD genes in this study were located in the Tartary Buckwheat Genome Project (TBGP; http://www.mbkbase.org/Pinku1/). The Tartary buckwheat accessions (XIQIAO; accessions number: CHUAN 2008013) materials used in the experiment were supplied by Professor Wang Anhu of Xichang University. The datasets supporting the conclusions of this article are included with in the article and its Supplementary files.

\section{Ethics approval and consent to participate}

The tartary buckwheat accessions (XIQIAO) materials used in the experiment were supplied by Professor Wang Anhu of Xichang University. These plant materials are widely used all over the world. and no permits are required for the collection of plant samples. The plant materials are maintained in accordance with the institutional guidelines of the College of Life Sciences, Sichuan Agricultural University, China. This article did not contain any studies with human participants or animals, and did not involve any endangered or protected species.

\section{Consent for publication}

Not applicable.

\section{Competing interests}

The authors declare that they have no competing interests.

\section{Author details}

${ }^{1}$ College of Life Science, Sichuan Agricultural University, Ya'an, China. ${ }^{2}$ School of Agriculture and Biolog, Shanghai Jiao Tong University, Shanghai, China.

Received: 14 November 2018 Accepted: 15 May 2019

Published online: 11 June 2019

\section{References}

1. Glazebrook J. Genes controlling expression of defense responses in Arabidopsis - 2001 status. Curr Opin Plant Biol. 2001;4(4):301-8.

2. Singh K, Foley RC, Oñate-Sánchez L. Transcription factors in plant defense and stress responses. Curr Opin Plant Biol. 2002;5(5):430-6.

3. Bürglin TR. A Caenorhabditis elegans prospero homologue defines a novel domain. Trends Biochem Sci. 1994;19(2):70-1.

4. Gehring WJ, M Affolter A, Burglin T. Homeodomain Proteins. Annu Rev Biochem. 1994;63(63):487.

5. Wolberger C. Homeodomain interactions. Curr Opin Struct Biol. 1996;6(1): 62-8.

6. Ariel FD, Manavella PA, Dezar CA, Chan RL. The true story of the HD-zip family. Trends Plant Sci. 2007;12(9):419-26.

7. Kawagashira N, Ohtomo Y, Murakami K, Matsubara K, Kawai J, Carninci P, Hayashizaki Y, Kikuchi S, Higo K: Multiple Zinc Finger Motifs with Comparison of Plant and Insect 2001(12):368-369.

8. Krishna SS, Majumdar I, Grishin NV. Structural classification of zinc fingers: survey and summary. Nucleic Acids Res. 2003;31(2):532-50.

9. Takatsuji H. Zinc-finger proteins: the classical zinc finger emerges in contemporary plant science. Plant Mol Biol. 1999;39(6):1073-8.

10. Mackay JP, Crossley M. Zinc fingers are sticking together. Trends Biochem Sci. 1998;23(1):1-4.

11. Windhövel A, Hein I, Dabrowa R, Stockhaus J. Characterization of a novel class of plant homeodomain proteins that bind to the C4 phosphoenolpyruvate carboxylase gene of Flaveria trinervia. Plant Mol Biol. 2001;45(2):201-14.

12. Hu W, Depamphilis CW, Ma H. Phylogenetic analysis of the plant-specific zinc finger-Homeobox and mini zinc finger gene families. J Integr Plant Biol. 2008;50(8):1031-45.

13. Ma J, Zheng L, Zhao C, Li G, Shen Y, An N, Zhang D, Han M. Genome-wide identification and expression analysis of half-size ABCG genes in Malus $\times$ domestica. Horticultural Plant Journal. 2018;4(2):45-54.

14. Khatun K, Nath UK, Robin AHK, Park JI, Lee DJ, Kim MB, Chang KK, Lim KB, Nou IS, Chung MY. Genome-wide analysis and expression profiling of zinc finger homeodomain ( ZHD) family genes reveal likely roles in organ development and stress responses in tomato. BMC Genomics. 2017;18(1): 695.

15. Hao W, Xiangjing Y, Xiaoqin L, Li W, Yi Z, Xiaozhao X, Yucheng Z, Xiping W. Genome-wide identification, evolution and expression analysis of the grape (Vitis vinifera L.) zinc finger-homeodomain gene family. Int J Mol Sci. 2014; 15(4):5730-48.

16. Wei $H$, Hong M. Characterization of a novel putative zinc finger gene MIF1: involvement in multiple hormonal regulation of Arabidopsis development. Plant J. 2010:45(3):399-422.

17. HC P, ML K, SM L, JD B, DJ Y, CO L, JC H, SY L, MJ C, WS C. Pathogeninduced binding of the soybean zinc finger homeodomain proteins GmZFHD1 and GmZF-HD2 to two repeats of ATTA homeodomain binding site in the calmodulin isoform 4 (GmCaM4) promoter. Nucleic Acids Res. 2007; 35(11):3612-23.

18. Nina F, Janko R, Iztok Joze K, Zhuanhua W, Zheng Z, Ivan K. Tartary buckwheat (Fagopyrum tataricum Gaertn.) as a source of dietary rutin and quercitrin. Journal of Agricultural \& Food Chemistry. 2003;51(22):6452.

19. QF C: Buckwheat plant science: Beijing, China: Science Press; 2012.

20. Bueso E, Muñoz-Bertomeu J, Campos F, Brunaud V, Martinez L, Sayas E, Ballester P, Yenush L, Serrano R. ARABIDOPSIS THALIANA HOMEOBOX25 uncovers a role for gibberellins in seed longevity. Plant Physiol. 2014;164(2): 999-1010.

21. Figueiredo D, Barros P, Cordeiro A, Serra T, Lourenço T, Chander S, Oliveira $M$, Saibo N. Seven zinc-finger transcription factors are novel regulators of the stress responsive gene OsDREB1B. J Exp Bot. 2012;63(10):3643-56. 
22. Liu M, Ma Z, Wang A, Zheng T, Huang L, Sun W, Zhang Y, Jin W, Zhan J, Cai $Y$, et al. Genome-wide investigation of the auxin response factor gene family in Tartary buckwheat (Fagopyrum tataricum). Int J Mol Sci. 2018; 19(11):3526.

23. Liu M, Fu Q, Ma Z, Sun W, Huang L, Wu Q, Tang Z, Bu T, Li C, Chen H: Genome-wide investigation of the MADS gene family and dehulling genes in tartary buckwheat (Fagopyrum tataricum). 2019.

24. Liu M, Ma Z, Sun W, Huang L, Wu Q, Tang Z, Bu T, Li C, Chen H: Genomewide analysis of the NAC transcription factor family in Tartary buckwheat (Fagopyrum tataricum). 2019, 20.

25. Liu M, Sun W, Ma Z, Zheng T, Huang L, Wu Q, Zhao G, Tang Z, Bu T, Li C, et al. Genome-wide investigation of the AP2/ERF gene family in tartary buckwheat (Fagopyum Tataricum); 2019. p. 19.

26. Kong X, Wei L, Jiang S, Dan Z, Cai G, Pan J, Li D: Genome-wide identification and expression analysis of calcium-dependent protein kinase in maize. BMC Genomics 2013, 14(1):433-433.

27. Liu M, Ma Z, Zheng T, Sun W, Zhang Y, Jin W, Zhan J, Cai Y, Tang Y, Wu Q: Insights into the correlation between physiological changes in and seed development of tartary buckwheat (Fagopyrum tataricum Gaertn ) BmC Genomics 2018, 19(1):648

28. TJ V, DG B, SD T. The origins of genomic duplications in Arabidopsis. Science. 2000:290(5499):2114-7.

29. Blanc $\mathrm{G}$, Hokamp K, Wolfe $\mathrm{KH}$. A recent polyploidy superimposed on older largescale duplications in the Arabidopsis genome. Genome Res. 2003;13(2):137.

30. Hongzhi K, Landherr LL, Frohlich MW, Jim LM, Hong M, Depamphilis CW Patterns of gene duplication in the plant SKP1 gene family in angiosperms: evidence for multiple mechanisms of rapid gene birth. Plant J. 2010;50(5): 873-85.

31. Cannon SB, Mitra A, Baumgarten A, Young ND, May G. The roles of segmental and tandem gene duplication in the evolution of large gene families in Arabidopsis thaliana. BMC Plant Biol. 2004;4(1):10.

32. Guixia X, Chunce G, Hongyan S, Hongzhi K. Divergence of duplicate genes in exon-intron structure. Proc Natl Acad Sci U S A. 2012;109(4):1187-92.

33. Filiz E, Tombuloğlu H. Genome-wide distribution of superoxide dismutase (SOD) gene families in Sorghum bicolor. Turk J Biol. 2015:39(1):49-59.

34. Wang W, Peng W, Ying L, Hou XL. Genome-wide analysis and expression patterns of ZF-HD transcription factors under different developmental tissues and abiotic stresses in Chinese cabbage. Mol Genet Genomics. 2016; 291(3):1451-64.

35. Zhang L, Li X, Ma B, Gao Q, Du H, Han Y, Li Y, Cao Y, Qi M, Zhu Y, et al. The Tartary Buckwheat Genome Provides Insights into Rutin Biosynthesis and Abiotic Stress Tolerance, vol. 10; 2017. p. 1224-37.

36. Tan $\mathrm{Q}$, Irish $\mathrm{V}$. The Arabidopsis zinc finger-homeodomain genes encode proteins with unique biochemical properties that are coordinately expressed during floral development. Plant Physiol. 2006;140(3):1095

37. Chojecki AJS, Bayliss MW, Gale MD. Cell production and DNA accumulation in the wheat endosperm, and their association with grain weight. Ann Bot 1986;58(6):809-17.

38. CHOJECKI AJS, GALE MD, BAYLISS MW. The number and sizes of starch granules in the wheat endosperm, and their association with grain weight. Ann Bot. 1986;58(6):819-31.

39. Munier-Jolain NG, Ney B. Seed growth rate in grain legumes II. Seed growth rate depends on cotyledon cell number. J Exp Bot. 1998;49(329):1971-6.

40. Sundaresan V. Control of seed size in plants. Proc Natl Acad Sci U S A. 2005; 102(50):17887-8

41. Mizutani M, Naganuma T, Tsutsumi K, Saitoh Y. The syncytium-specific expression of the Orysa;KRP3 CDK inhibitor: implication of its involvement in the cell cycle control in the rice (Oryza sativa L.) syncytial endosperm. J Exp Bot. 2010;61(3):791.

42. H W, L B, U W. Molecular physiology of legume seed development. Annu Rev Plant Biol. 2004:56(56):253-79.

43. Liu M, Ma Z, Zheng T, Wang J, Huang L, Sun W, Zhang Y, Jin W, Zhan J, Cai $Y$, et al. The potential role of auxin and abscisic acid balance and FtARF2 in the final size determination of Tartary buckwheat fruit. Int J Mol Sci. 2018; 19(9):2755.

44. Guilfoyle T, Hagen G, Ulmasov T, Murfett J. How does auxin turn on genes? Plant Physiol. 1998;118(2):341-7.

\section{Publisher's Note}

Springer Nature remains neutral with regard to jurisdictional claims in published maps and institutional affiliations.

\section{Ready to submit your research? Choose BMC and benefit from:}

- fast, convenient online submission

- thorough peer review by experienced researchers in your field

- rapid publication on acceptance

- support for research data, including large and complex data types

- gold Open Access which fosters wider collaboration and increased citations

- maximum visibility for your research: over $100 \mathrm{M}$ website views per year

At BMC, research is always in progress.

Learn more biomedcentral.com/submissions 